\title{
Equidecomposability, volume formulae and orthospectra
}

\author{
HideTOSHI MASAI \\ GREG MCSHANE
}

\begin{abstract}
Bridgeman-Kahn and Calegari derived formulae for the volumes of compact hyperbolic $n$-manifolds with totally geodesic boundary in terms of the orthospectrum. Their methods are apparently different from each other, and involve computing the volume of different subspaces of unit tangent bundle of hyperbolic $n$-space. In this paper, we show that the two volume formulae coincide. We also derive a closed form of the formula in dimension 3.
\end{abstract}

57M50; 32Q45

\section{Introduction}

Bridgeman-Kahn and Calegari derived formulae for the volumes of compact hyperbolic $n$-manifolds with totally geodesic boundary in terms of the orthospectrum of the manifold. Both methods for producing the formulae are based on decomposing the unit tangent bundle into countably many pieces, each of which is naturally associated to a unique orthogeodesic. In fact, each of these pieces is congruent to a model piece, respectively $\mathcal{B}(l)$ for the Bridgeman-Kahn decomposition and $\mathcal{C}(l)$ for Calegari's, determined up to isometry by the length $l$ of the corresponding orthogeodesic $\alpha^{*}$. So the volume of the unit tangent bundle can be expressed as a sum of the volumes of these pieces and each volume only depends on the length of an orthogeodesic. The formulae obtained are valid for all compact hyperbolic $n$-manifolds with totally geodesic boundary, however, the decompositions used by Bridgeman-Kahn and Calegari are quite different. It is natural to ask how the terms in the two formula are related. We show that the two formulae coincide, that is, for each orthogeodesic the associated Bridgeman-Kahn model piece and the Calegari model piece have the same volume regardless of the dimension. Throughout $\operatorname{vol}_{n}$ will denote the volume of an $n$-dimensional orientable manifold.

Theorem 1 For all $n \geq 2$,

$$
\operatorname{vol}_{2 n-1}(\mathcal{B}(l))=\operatorname{vol}_{2 n-1}(\mathcal{C}(l)) .
$$


We note that for $n=2$, Calegari [4] obtained this result by direct computation. Our method is geometric, we will show that the pair of sets $\mathcal{B}(l)$ and $\mathcal{C}(l)$ satisfy a property which we call countable equidecomposability (Section 6). This generalises the familiar notion of scissors congruence by allowing decompositions having countably many pieces rather than just finitely many.

Both Bridgeman and Calegari found a closed expressions for the volume of the 2-dimensional pieces and they give integral formulae for $\operatorname{vol}_{2 n-1}(\mathcal{B}(l))$ and $\operatorname{vol}_{2 n-1}(\mathcal{C}(l))$ respectively in all dimensions $n \geq 2$. When $n$ is odd and in particular in dimension 3 , Calegari's decomposition is more convenient for purposes of calculation. We exploit this to give a closed form for the volume of the pieces in terms of an ortholength $l$.

\section{Theorem 2}

$$
\operatorname{vol}_{5}(\mathcal{C}(l))=\frac{2 \pi(l+1)}{e^{2 l}-1}
$$

In two dimensions the volume of each piece turns out to be the Rogers' dilogarithm of a simple function of the ortholength; see Bridgeman [2] and [4]. This case is of particular interest since the deformation theory of convex surfaces leads yields functional relations for the dilogarithm. However, as one sees from the formula above, in three dimensions the volume of each piece can be written in terms of the ortholength and its exponential. The deformation theory of hyperbolic 3-manifolds which have totally geodesic boundary is trivial and no functional relations are to be expected.

More on closed forms for the volume of pieces Calegari has also used his method to compute an expression for the terms in the 3-dimensional Basmajian identity [1]. In particular, for a compact hyperbolic 3-manifold $M$ with totally geodesic boundary $\partial M$, one has

$$
-\chi(\partial M)=\sum_{\alpha^{*}} \frac{4}{e^{\ell\left(2 \alpha^{*}\right)}-1},
$$

where the sum is over all orthogeodesics $\alpha^{*}$. Compare this with Bridgeman-Kahn:

$$
2 \operatorname{vol}_{3}(M)=\sum_{\alpha^{*}} \frac{\ell\left(\alpha^{*}\right)+1}{e^{2 \ell\left(\alpha^{*}\right)}-1} .
$$

Thus both the volume of the 3-manifold and the area of the boundary are determined by the orthospectrum. Moreover, these quantities are written as series over the orthospectra and the terms are expressed using just standard functions. 
Whilst in all dimensions $n \geq 2$ the term in Basmajian's identity is readily computable in terms of standard functions (see [4]), there is a curious dichotomy for the BridgemanKahn and Calegari identities which we will explain briefly. Both Bridgeman-Kahn and Calegari give integral formulae for the terms in the series that yields the volume of the hyperbolic $n$-manifold. Bridgeman and Kahn note further that, when $n$ is even, it is possible to find a closed expression for this term involving usual functions and the dilogarithm [3, Corollary 8]. On the other hand, Calegari gives an integral expression which readily yields a closed expression for the terms, again involving usual functions and the dilogarithm, but only when $n$ is odd (Proposition 6 below). By Theorem 1 Bridgeman-Kahn's and Calegari's terms are identical so this situation is somewhat puzzling and we can offer no explanation.

Acknowledgements The authors thank Martin Bridgeman, Danny Calegari and Sadayoshi Kojima for many helpful conversations and remarks. This work was carried out while the second author was visiting Tokyo Tech in the summer of 2012 and, as such, he is indebted to Professor S Kojima for his kindness and hospitality. The work of the first author was supported by JSPS Research Fellowship for Young Scientists.

\section{Preliminaries}

\subsection{Orthogeodesics and the orthospectrum}

We consider compact hyperbolic manifolds $M$ of finite volume with nonempty totally geodesic boundary $\partial M$. Such a manifold is obtained as the quotient of a convex subset of $\mathbb{H}^{n}$ by a group of orientation preserving isometries $\Gamma$. The limit set $\Lambda$ of $\Gamma$ is a nonempty $\Gamma$-invariant closed subset of $\partial \mathbb{H}^{n}$ which, by a theorem of Ahlfors, is measure 0 . Henceforth, we identify $M$ with the quotient of the convex hull of $\Lambda$ by $\Gamma$.

The complement of $\Lambda$ consists of countably many $(n-1)$-dimensional balls and the convex hull of each ball is a half space bounded by a totally geodesic $(n-1)-$ dimensional hyperplane which we call a side of the convex hull. To each side $D_{a}$ there is a subgroup $\Gamma_{D_{a}}<\Gamma$ consisting of all the elements such that $g\left(D_{a}\right)=D_{a}$. The quotient of the side by $\Gamma_{D_{a}}$ is a compact, totally geodesic, $(n-1)$-dimensional manifold embedded in the boundary of $\partial M$.

Let $D_{a}, D_{b}$ be a pair of sides then the orthogeodesic connecting $D_{a}$ and $D_{b}$ is the shortest geodesic arc $\hat{\alpha}^{*}$ with an endpoint in $D_{a}$ and the other in $D_{b}$. The length of the orthogeodesic $\ell\left(\widehat{\alpha}^{*}\right)$ is the minimum distance between $D_{a}$ and $D_{b}$. If $\hat{\alpha}^{*}$ is the orthogeodesic joining a pair of sides of the convex hull then its image $\alpha^{*}$ in the quotient $M$ is an orthogeodesic of length $\ell\left(\hat{\alpha}^{*}\right)$ joining a pair of totally geodesic 
boundary components. We call the set of ortholengths $\ell\left(\alpha^{*}\right)$ (with multiplicities in $M$ ) the orthospectrum of $M$. Less formally the orthospectrum of the manifold $M$ is the set of lengths of common perpendiculars between (not necessarily distinct) boundary components.

\subsection{The unit tangent bundle}

Our arguments are based on elementary hyperbolic geometry. We shall use the following (standard) notation throughout.

We denote $p: T \mathbb{H}^{n} \rightarrow \mathbb{H}^{n}$ the canonical map that associates to a tangent vector its basepoint. Let $A$ be an isometry (diffeomorphism) of $\mathbb{H}^{n}$ then it induces a diffeomorphism of the tangent bundle which we continue to denote by $A$.

If $v \in T \mathbb{H}^{n}$ is a (nonzero) tangent vector then

$$
\gamma_{v}: \mathbb{R} \rightarrow \mathbb{H}^{n}
$$

is the unique geodesic parameterised by arc length such that $\dot{\gamma}_{v}(0)$ is a positive multiple of $v$. The geodesic $\gamma_{v}$ determines a pair of distinct points $\gamma_{v}( \pm \infty)$ in the ideal boundary of $\mathbb{H}^{n}$. Observe that the map

$$
\begin{aligned}
v & \mapsto \gamma_{v}(-\infty), \\
T \mathbb{H}^{n} & \rightarrow \partial \mathbb{H}^{n},
\end{aligned}
$$

is continuous and, in particular, the preimage of any measurable subset of $\partial \mathbb{H}^{n}$ is a measurable subset of the tangent bundle.

Whenever we speak of a geodesic $\hat{\alpha}$ in $\mathbb{H}^{n} \cup \partial \mathbb{H}^{n}$ we mean the union of a geodesic $\hat{\alpha}$ and its ideal endpoints $\alpha^{ \pm}$.

As discussed in [3], the unit tangent bundle $T_{1} \mathbb{H}^{n}$ has a standard volume form $\Omega$, which is just the product of the standard volume forms on $\mathbb{H}^{n}$ and $S^{n-1}$. To obtain an explicit formula for $d \Omega$, it is convenient to try to parametrize unit tangent vectors by triples

$$
(x, y, t) \in \mathbb{R}^{n-1} \times \mathbb{R}^{n-1} \times \mathbb{R} .
$$

We consider the upper half space model of $\mathbb{H}^{n}$ so that the ideal boundary is identified with $\mathbb{R}^{n-1} \cup\{\infty\}$. A point $v \in T_{1} \mathbb{H}^{n}$ determines a unique directed geodesic $\gamma_{v}$ and so an ordered pair of points $\left(\gamma_{v}(-\infty), \gamma_{v}(\infty)\right)$ in the ideal boundary $\mathbb{R}^{n-1} \cup\{\infty\}$ and, provided neither of these points is $\infty$, we may set $(x, y)=\left(\gamma_{v}(-\infty), \gamma_{v}(\infty)\right)$. The last coordinate $t \in \mathbb{R}$ is the signed hyperbolic length between the highest point of $\gamma_{v}$ and $p(v)$. Our parametrization is defined on a open dense subset of $T_{1} \mathbb{H}^{n}$ and it is 
easy to check that the complement has measure zero so we may ignore its contribution when we compute volumes in $T_{1} \mathbb{H}^{n}$. With this parametrization, we have

$$
d \Omega=\frac{2 d V(x) d V(y) d t}{|x-y|^{2 n-2}},
$$

where $d V(x)=d x_{1} d x_{2} \cdots d x_{n-1}$ for $x=\left(x_{1}, x_{2}, \ldots, x_{n}\right) \in \mathbb{R}^{n-1}$.

\section{Subsets of the tangent bundle}

Let $\widehat{\delta}$ and $\hat{\alpha}$ be a pair of disjoint geodesics in the $\mathbb{H} \cup \partial \mathbb{H}$. The convex hull of $\widehat{\delta}$ and $\widehat{\alpha}$ is an ideal quadrilateral $\mathcal{Q}$ (see figure). The problem is to show that a certain pair of subsets of the unit tangent bundle of $\mathbb{H}$, have the same volume.

- Bridgeman's set $\mathcal{B}(l)$ is the set of unit vectors $v$ tangent to geodesic segments joining $\widehat{\alpha}$ to $\widehat{\delta}$. More formally, it is the set of $v \in p^{-1}(\mathcal{Q})$ satisfying

(i) the ray $\gamma_{v}\left(\mathbb{R}_{+}\right)$meets $\hat{\delta}$,

(ii) the ray $\gamma_{v}\left(\mathbb{R}_{-}\right)$meets $\widehat{\alpha}$.

We shall call this set the set of bridging vectors.

- Calegari's set $\mathcal{C}(l)$ is the set of unit vectors $v$ such that

(i) the ray $\gamma_{v}\left(\mathbb{R}_{+}\right)$meets $\hat{\delta}$,

(ii) the point $p(v)$ is in the chimney (see below) of the quadrilateral $\mathcal{Q}$.

The chimney is the dark subset of the ideal quadrilateral in Figure 1 it is the convex hull of $\hat{\alpha} \cup\left\{\alpha^{+}, \alpha^{-}\right\}$and the nearest point retraction of $\hat{\alpha}$ to $\hat{\delta}$. Following Calegari, we say that $\hat{\alpha}$ is the top of the chimney and the nearest point retraction of $\hat{\alpha}$ to $\hat{\delta}$ is its base which we will denote by $B$. The chimney is a convex quadrilateral with the top and the base forming a pair of sides and we refer to the remaining pair of sides as the walls.

The top of the chimney bounds an interval $T_{\infty}=\left[\alpha^{+}, \alpha^{-}\right] \subset \mathbb{R}$ and it is easy to see that the nearest point retraction

$$
\pi_{\widehat{\delta}}: T_{\infty} \rightarrow \widehat{\delta}
$$

is a homeomorphism.

Our Theorem 1 says that the sets $\mathcal{B}(l), \mathcal{C}(l)$ have the same volume. We will begin by discussing in detail the case $n=2$ in the next section (compare Calegari in [5].) 


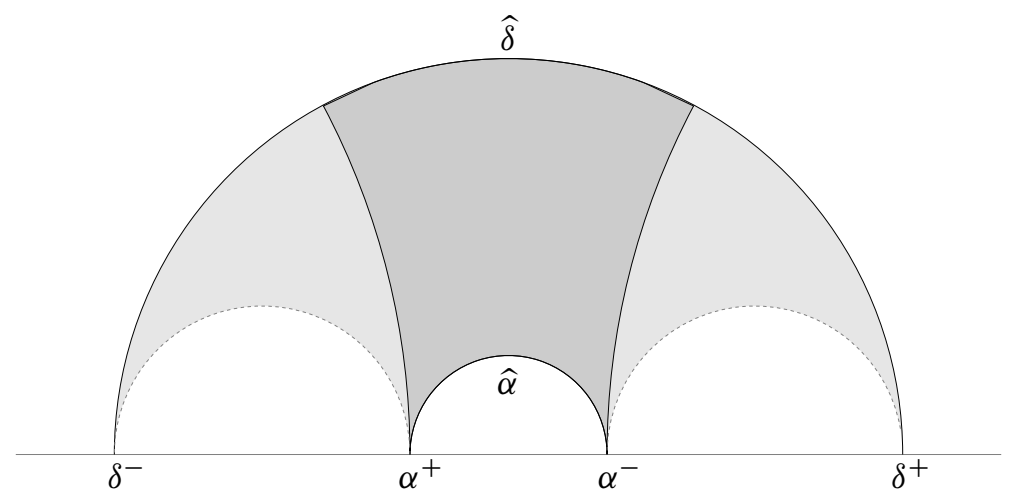

Figure 1: The quadrilateral $\mathcal{Q}$ and its chimney

\section{Comparing the sets $\mathcal{B}(l), \mathcal{C}(l)$}

Calegari [4] defines a positive function $h: \Delta \rightarrow \mathbb{R}^{+}$on the chimney whose value is the density in the unit tangent sphere at a point $x$ of vectors $v \in \mathcal{C}(l)$. Thus, by Fubini's theorem, the volume of $\mathcal{C}(l)$ is the integral of $h$ over the chimney $\Delta$. In two dimensions the value of $h(x)$ is the visual measure of $\hat{\delta}$ at $x$, and this is just the angle between the pair of geodesics passing through $x$ and the ideal points $\delta^{ \pm}$(Figure 2).

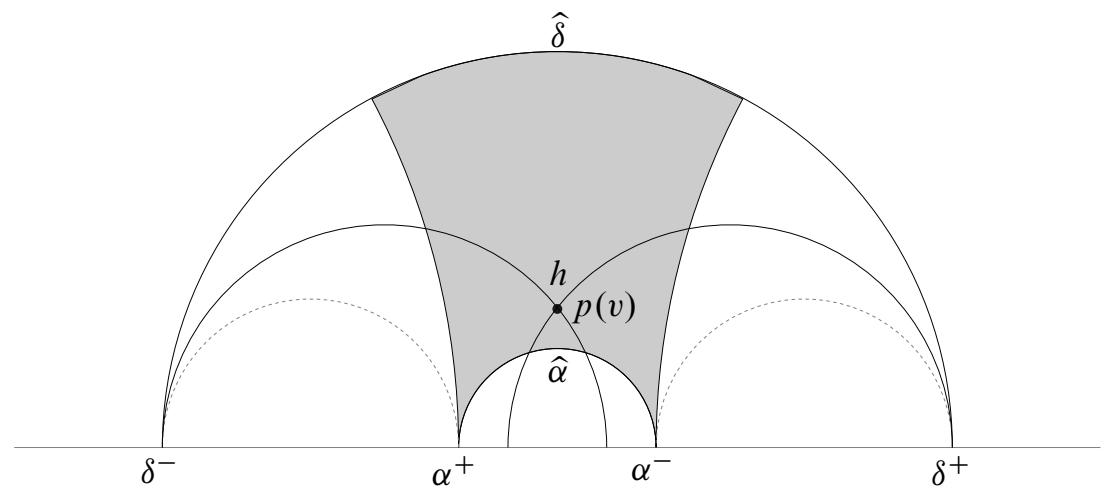

Figure 2: Calegari's angle

To see that the sets $\mathcal{B}(l), \mathcal{C}(l)$ have the same volume we show how one can associate to a vector in Calegari's set $\mathcal{C}(l)$ a unique vector tangent to one of Bridgeman's geodesic arcs: more formally that there is a measure preserving bijection $\phi$ between a subset of full measure of Bridgeman's set and a subset of full measure of Calegari's set. 
In the next figure we see one of Bridgeman's arcs $\gamma$ that passes through $p(v)$ and exits the chimney by a side. By the uniqueness of geodesics in $\mathbb{H}$, that the tangent vector to $\gamma$ at $x \in \gamma \cap$ chimney is in $\mathcal{C}(l)$; so on this portion we can take $\phi$ to be the identity. The problem is thus to define such a map for vectors $v$ tangent to $\gamma$ at points $p(v)$ in the complement of the chimney.

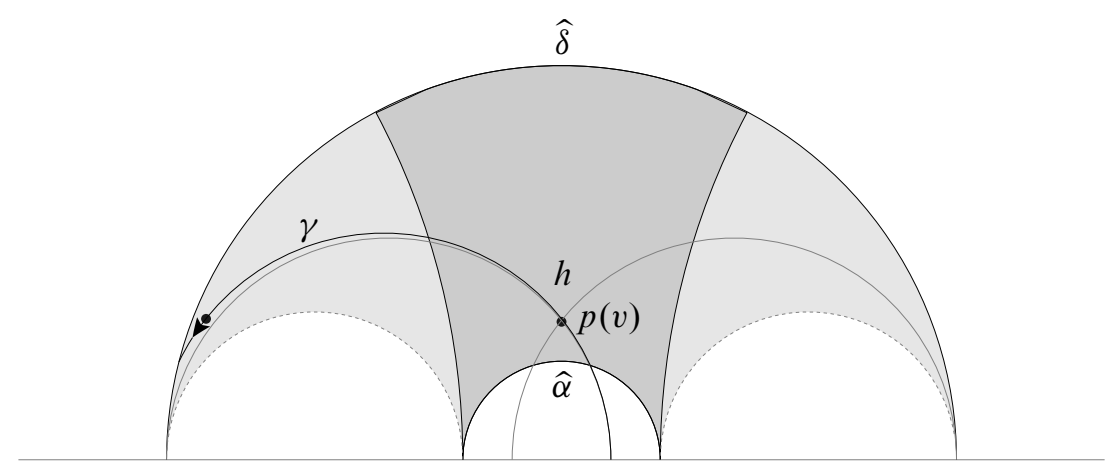

Figure 3: How do I account for all $v$ tangent to the geodesic $\gamma$ ?

\section{Constructing a pair of measurable bijections}

We begin by covering by the quadrilateral with copies of the chimney. Let $A$ denote the side pairing that takes one of the walls of the chimney to the other. We may suppose that the attracting fixed point of $A$ is $\delta^{+}$and the repelling fixed point $\delta^{-}$. Note that the isometry $A$ is not an element of the covering group $\Gamma$.

Now we have a cover of the quadrilateral by translates of the chimney

$$
\mathcal{Q} \subset \bigcup_{k} A^{k} \text { (chimney); }
$$

see Figure 4. If $x=p(v)$ is a point of $\gamma$ then (up to a set of measure 0) there is a unique power of $A$ such that $A^{n}(x)$ is in the chimney. Since $A$ preserves $\widehat{\delta}$, we have $A^{n}(\gamma) \cap \hat{\delta} \neq \varnothing$, so the image of $v$ by $A^{n}$ is tangent to a geodesic, namely $A^{n}(\gamma)$, that exits via $\widehat{\delta}$. This of course means that $A^{n}(v)$ is in Calegari's set $\mathcal{C}(l)$.

We define a map

$$
\begin{aligned}
& f: v \mapsto A^{n}(v), \quad p(v) \in A^{-n} \text { (chimney), } \\
& \mathcal{B}(l) \rightarrow \text { tangent vectors to chimney. }
\end{aligned}
$$




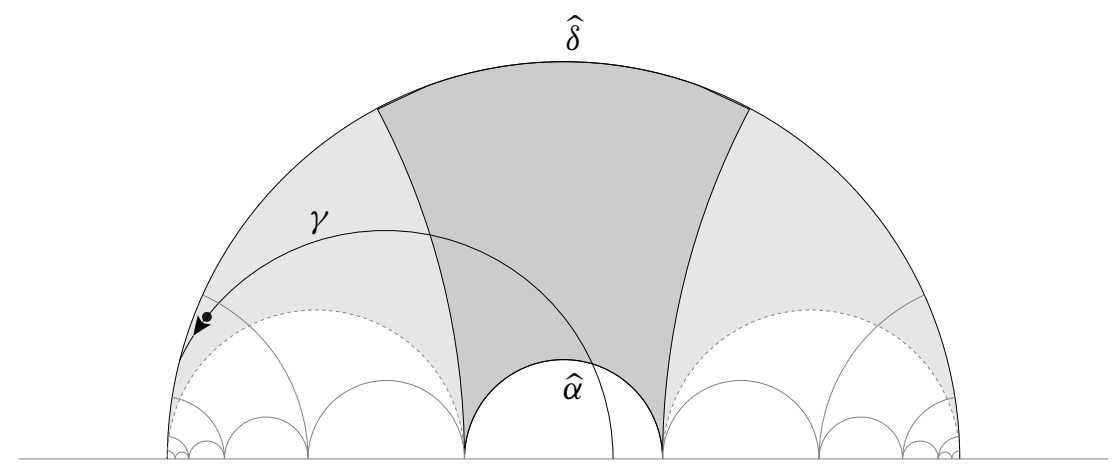

Figure 4: Covering using the chimney, the point $x=p(v)$ is marked on the left

Note that, for each $k \in \mathbb{Z}$, the set $p^{-1}\left(A^{k}\right.$ (chimney)) is a measurable subset of the tangent bundle so the map is

- well-defined on a subset of full measure in $\mathcal{B}(l)$,

- measure-preserving where it is defined since $A$ is an isometry.

To show that it is a bijection (on a set of full measure) we construct an (almost everywhere defined) inverse. Let $v$ be a vector in $\mathcal{C}(l)$, by definition $\gamma_{v}\left(\mathbb{R}_{+}\right)$meets $\hat{\delta}$, and consider the ideal point $\gamma_{v}(-\infty)$. Since geodesics meet at most once this is a point of the interval $] \delta^{-}, \delta^{+}\left[\right.$. This interval is tessellated by the intervals $A^{k}\left(\left[\alpha^{+}, \alpha^{-}\right]\right)$so one can define a map

$$
\begin{aligned}
& \phi: v \mapsto A^{n}(v), \text { if } \gamma_{v}(-\infty) \in A^{-n}(] \alpha^{+}, \alpha^{-}[), \\
& \mathcal{C}(l) \rightarrow \text { tangent vectors to the ideal quadrilateral } \mathcal{Q} .
\end{aligned}
$$

Since $v$ is tangent to a geodesic that joins $\widehat{\delta}$ to $A^{-n}(\widehat{\alpha})$, its image $\phi(v)$ is tangent to a vector that joins $\widehat{\delta}$ to $\widehat{\alpha}$.

\subsection{Inverses}

It is easy to check that these maps are inverses by examining the last figure.

- The map $f$ breaks our geodesic arc $\gamma$ into 3 pieces by translation by $A$ and intersection with the chimney.

- $\phi$ takes each of these pieces, extends it to find an ideal endpoint in the interval ]$\delta^{-}, \delta^{+}[\subset \mathbb{R}$, then glues them back using this data to make the original arc. 


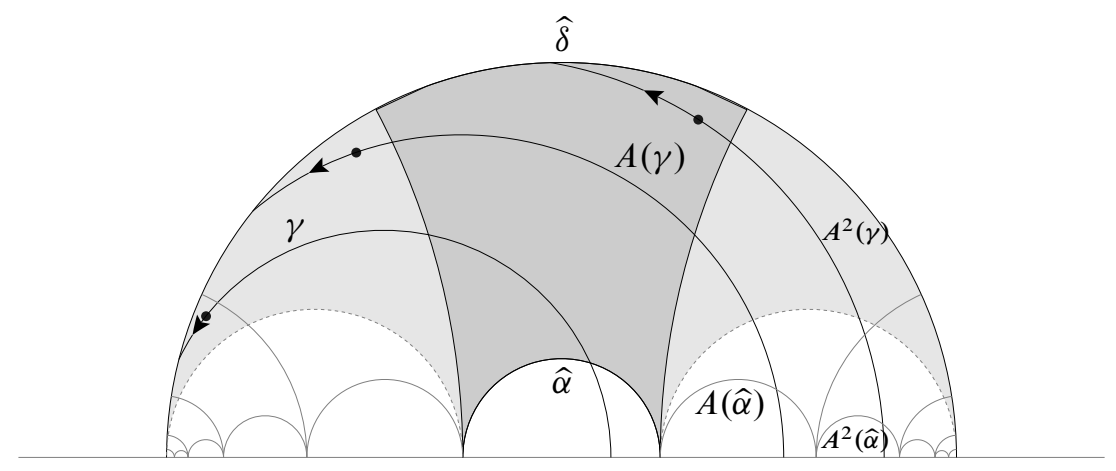

Figure 5: Translates of the geodesic $\gamma$ and the point $p(v)$

\section{Equidecomposability and volume}

A more succinct way of thinking of this is that $\mathcal{B}(l)$ and $\mathcal{C}(l)$ contain sets of full measure that are countable equidecomposable (we define this below). Recall that a pair of subsets $X, Y$ of a metric space are equidecomposable if there exists a pair of decompositions

$$
X=\bigsqcup_{k=0}^{N} X_{k}, \quad Y=\bigsqcup_{k=0}^{N} Y_{k},
$$

and isometries $\phi_{k}$ such that $Y_{k}=\phi_{k}\left(X_{k}\right)$.

We extend this definition slightly and say that subset $X, Y$ of a metric space are countable equidecomposable if there exists a pair of decompositions

$$
X=\bigsqcup_{k=0}^{\infty} X_{k}, \quad Y=\bigsqcup_{k=0}^{\infty} Y_{k},
$$

and isometries $\phi_{k}$ such that

$$
Y_{k}=\phi_{k}\left(X_{k}\right) \text {. }
$$

If the metric space is equipped with a countably additive measure $\mu$, such that the isometries are measure preserving maps, then we conclude that $X$ and $Y$ have the same $\mu$-volume provided the pieces $X_{k}, Y_{k}$ are measurable.

\subsection{Equidecomposability in dimension 2}

Using this notion we reformulate the ideas of the previous section.

Theorem 3 Let $\Delta \subset \mathcal{Q}$ be a chimney and $\dot{\Delta}$ its interior. Then there are subsets of full measure $X \subset \mathcal{B}(l)$ and $Y \subset \mathcal{C}(l)$ that are countable equidecomposable. 
Proof Define

$$
\begin{aligned}
X_{k} & :=\left\{v \in \mathcal{B}(l), p(v) \in A^{-k}(\dot{\Delta})\right\}, \\
Y_{k} & :=\left\{v \in \mathcal{C}(l), \gamma_{v}\left(\mathbb{R}_{-}\right) \cap A^{k}(\widehat{\alpha}) \neq \varnothing\right\} .
\end{aligned}
$$

It is easy to see that the sets

$$
X=\bigsqcup X_{k}, \quad Y=\bigsqcup Y_{k},
$$

are full measure in $\mathcal{B}(l)$ and $\mathcal{C}(l)$ respectively.

Finally we show that $\phi_{k}=A^{k}$. Suppose $v \in \mathcal{B}(l)$ then $\gamma_{v}\left(\mathbb{R}_{-}\right) \cap \hat{\alpha} \neq \varnothing$ so that $A^{k}(v)$ satisfies

$$
\gamma_{A^{k}(v)}\left(\mathbb{R}_{-}\right) \cap A^{k}(\widehat{\alpha}) \neq \varnothing
$$

Further, if $v \in X_{k}$ then

$$
p\left(A^{k}(v)\right)=A^{k}(p(v)) \in \dot{\Delta}
$$

From (1) and (2) one has $A^{k}\left(X_{k}\right) \subset Y_{k}$. The other inclusion follows by a similar argument.

\subsection{Equidecomposability from covers}

In fact, to construct appropriate decompositions $X_{i}, Y_{i}$, one only really needs a countable cover of the interval $] \delta^{-}, \delta^{+}$[ by $A$-translates of $T_{\infty}=\left[\alpha^{+}, \alpha^{-}\right]$. Note that such a cover determines a countable cover of the geodesic $\hat{\delta}$ via nearest point retraction to $\hat{\delta}$.

Theorem 4 Let $A_{k}, A_{0}=I$ be hyperbolic isometries

(i) preserving the components of $\partial \mathbb{H} \backslash\left\{\delta^{+}, \delta^{-}\right\}$,

(ii) such that $\left\{A_{k}(B)\right\}_{k=0}^{\infty}$ cover $\widehat{\delta}$.

Then there are measurable sets $X_{k}, Y_{k}$ such that

$$
\mathcal{B}(l)=\bigsqcup_{k} X_{k}, \quad \mathcal{C}(l)=\bigsqcup_{k} Y_{k}
$$

and $A_{k}\left(X_{k}\right)=Y_{k}$, for all $k \geq 0$. 
Proof The base $B$ is the image under the nearest point retraction to $\hat{\delta}$ of a disc $T_{\infty} \subset \partial \mathbb{H}$. Since the $A_{k}(B)$ cover $\hat{\delta}$, the translates $\left\{A_{k}\left(T_{\infty}\right)\right\}_{k=0}^{\infty}$ cover the component of $\partial \mathbb{H} \backslash\left\{\delta^{+}, \delta^{-}\right\}$containing the endpoints $\gamma_{v}(-\infty), v \in \mathcal{C}(l)$. We define a map

$$
\begin{aligned}
\sigma: \mathcal{C}(l) & \rightarrow \mathbb{Z}^{+} \\
\sigma(v) & =\inf \left\{k \mid \gamma_{v}(-\infty) \in A_{k}\left(T_{\infty}\right)\right\}
\end{aligned}
$$

By construction $\sigma$ is such that $\sigma^{-1}(\{0, \ldots, k\})$ is precisely the union $\bigcup_{j=1}^{k} A_{k}\left(T_{\infty}\right)$ which is a measurable set. It follows that

$$
Y_{k}:=\sigma^{-1}(\{k\})=\sigma^{-1}(\{0, \ldots, k\}) \backslash \sigma^{-1}(\{0, \ldots, k-1\})
$$

is measurable too. Analogously we define the set $X_{k}$ to be $\tau^{-1}(\{k\})$ where

$$
\begin{aligned}
\tau: \mathcal{B}(l) & \rightarrow \mathbb{Z}^{+}, \\
\tau(v) & =\inf \left\{k \mid p(v) \in A_{k}^{-1}(\Delta)\right\} .
\end{aligned}
$$

Exactly same argument as before shows that $A_{k}\left(X_{k}\right)=Y_{k}$.

\section{Equidecomposability in higher dimensions}

Let $D_{b}$ and $D_{a}$ be a pair of disjoint totally geodesic hyperplanes $\mathbb{H}^{n}$ and consider the unit tangent bundle over their convex hull $\mathcal{Q}$. The walless chimney is the convex hull of $D_{a}$ and its image under nearest point retraction to $D_{b}$ which we still denote $B$. The top of the chimney is $D_{a}$ and its base is $B$. The top of the chimney bounds a round disc $T_{\infty} \subset \partial \mathbb{H}^{n}$ and the base $B$ is an open round ball.

The sets $\mathcal{B}(l)$ and $\mathcal{C}(l)$ are defined as before:

- $\mathcal{B}(l)$ is the set of unit tangent vectors $v$ such that

(i) the ray $\gamma_{v}\left(\mathbb{R}_{+}\right)$meets $D_{b}$,

(ii) the ray $\gamma_{v}\left(\mathbb{R}_{-}\right)$meets $D_{a}$.

- $\mathcal{C}(l)$ is the set of unit tangent vectors $v$ such that

(i) the ray $\gamma_{v}\left(\mathbb{R}_{+}\right)$meets $D_{b}$,

(ii) the point $p(v)$ is in the walless chimney.

If $n>2$ then the plane $D_{b}$ does not admit a tiling by copies of $B$. However, it does admit a covering by open discs congruent to $B$; for example choose any countable dense subset $P \subset D_{b}$ and take the cover by discs with centers in $P$ and radius equal to that of $B$. Any such cover yields a covering of $T_{\infty}$ by taking preimages under the nearest point retraction. 
Theorem 5 Let $A_{k}, A_{0}=I$ be hyperbolic isometries

(i) preserving the components of $\partial \mathbb{H} \backslash \partial D_{b}$,

(ii) such that $\left\{A_{k}(B)\right\}_{k=0}^{\infty}$ cover $D_{b}$.

Then there are measurable sets $X_{k}, Y_{k}$ such that

$$
\mathcal{B}(l)=\bigsqcup_{k} X_{k}, \quad \mathcal{C}(l)=\bigsqcup_{k} Y_{k}
$$

and $A_{k}\left(X_{k}\right)=Y_{k}$, for all $k \geq 0$.

Proof Note now that $D_{b}$ is a totally geodesic hyperplane, that is a copy of $\mathbb{H}^{n-1}$ embedded in $\mathbb{H}^{n}$, so that its boundary $\partial D_{b}$ is a round sphere in $\partial \mathbb{H}^{n}$. The sphere separates $\partial \mathbb{H}^{n}$ into two round balls, one of which is disjoint from $\Lambda$.

The base $B$ is the image under the nearest point retraction to $D_{b}$ of a disc $T_{\infty} \subset \partial \mathbb{H}^{n}$. Since the $A_{k}(B)$ cover $D_{b}$, then the translates $\left\{A_{k}\left(T_{\infty}\right)\right\}_{k=0}^{\infty}$ cover the component of $\partial \mathbb{H}^{n} \backslash \partial D_{b}$ whose closure contains the limit set and hence the endpoints $\gamma_{v}(-\infty), v \in \mathcal{C}(l)$.

The rest of the proof is as before.

\section{Three-dimensional case}

In this section we show that the volume of Calegari's piece for dimension 3 can be expressed in terms of the ortholength and its exponential. See also Fenchel [6] for elementary hyperbolic geometry.

\subsection{Integral formula for the volume of Calegari's piece}

Recall from [4] that $h: \mathcal{C}(l) \rightarrow \mathbb{R}_{+}$denotes Calegari's angle function (see Figure 2) and that $\operatorname{vol}_{2 n-1}(\mathcal{C}(l))$ is the integral of $h$ over a chimney $\Delta$. Consider the level sets and value of $h$ on the level sets. Let $C_{t}$ denote the subset of the chimney at distance $t$ from the base, it is easy to see that the value of $h$ is constant on $C_{t}$. We begin by discussing the geometry of these level sets and for this we need to study Lambert quadrilaterals. 
8.1.1 Lambert quadrilaterals Let $Q(\phi)$ be a Lambert quadrilateral, that is a hyperbolic quadrilateral with three right angles and an angle $\phi<\pi / 2$, (Figure 6). Observe that there is a pair of edges which have both endpoints at right angles. If $l$ and $\iota_{\phi}(l)$ denote the lengths of these edges then, from elementary hyperbolic geometry, we have

$$
\sinh \left(\iota_{\phi}(l)\right)=\cos (\phi) / \sinh (l) .
$$

Further, if $\phi=0$, then we write $\iota(l):=\iota_{0}(l)$, and we have the following equivalent formulae:

$$
\begin{aligned}
1 / \cosh ^{2}(l)+1 / \cosh ^{2}(\iota(l))=1 & \Leftrightarrow \cosh (\iota(l))=1 / \tanh (l) \\
& \Leftrightarrow \sinh (\iota(l))=1 / \sinh (l) .
\end{aligned}
$$

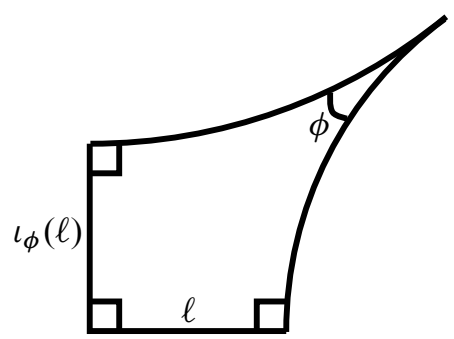

Figure 6: A Lambert quadrilateral

Now the nearest point retraction of $C_{t}$ to the base of the chimney is surjective if $t \leq l$, and otherwise the image is an annulus with outer radius $\iota(l)$, and inner radius $\iota_{\phi}(l)$, where $\phi$ is defined implicitly by $\sin (\phi)=\cosh (l) / \cosh (t)$. This latter formula in turn yields that $\cosh \left(\iota_{\phi}(l)\right)=\tanh (t) / \tanh (l)$ since

$$
\begin{aligned}
\cosh ^{2}\left(l_{\phi}(l)\right) & =\sinh ^{2}(l(l)) \cos ^{2}(\phi)+1 \\
& =\sinh ^{2}(l(l))\left(1-\left(\cosh ^{2}(l) / \cosh ^{2}(t)\right)\right)+1 \\
& =\frac{\cosh ^{2}(t)-\cosh ^{2}(l)+\sinh ^{2}(l) \cosh ^{2}(t)}{\sinh ^{2}(l) \cosh ^{2}(t)} \\
& =\frac{\cosh ^{2}(t)\left(1+\sinh ^{2}(l)\right)-\cosh ^{2}(l)}{\sinh ^{2}(l) \cosh ^{2}(t)} \\
& =\frac{\left(\cosh ^{2}(t)-1\right) \cosh ^{2}(l)}{\sinh ^{2}(l) \cosh ^{2}(l)} \\
& =\frac{\tanh ^{2}(t)}{\tanh ^{2}(l)} .
\end{aligned}
$$


8.1.2 Volumes of balls in hyperbolic or spherical space Let $V_{n}^{H}(r)\left(\right.$ resp. $\left.V_{n}^{S}(r)\right)$ denote the volume of a ball of radius $r$ in hyperbolic (resp. spherical) $n$-space. The following integral formula for $V_{n}^{H}$ and $V_{n}^{S}$ are well known (see Ratcliffe [7]):

$$
V_{n}^{H}(r)=\Omega_{n-1} \int_{0}^{r} \sinh ^{n-1}(t) d t, \quad V_{n}^{S}(r)=\Omega_{n-1} \int_{0}^{r} \sin ^{n-1}(t) d t,
$$

where $\Omega_{n-1}=2 \pi^{n / 2} / \Gamma(n / 2)$ is the volume of a Euclidean sphere of dimension $n-1$ and radius 1 .

8.1.3 Calegari's integral formula We now state the integral formula which will allow us to calculate $\operatorname{vol}_{5}(\mathcal{C}(l))$. Observe that, for $q \in \mathcal{C}(l), h(q)$ depends only on the distance $t$ between the base of chimney and $q$. Writing $h$ as a function of $t$ yields

$$
h(q)=\Omega_{n-1}^{-1} V_{n-1}^{S}(\arcsin (1 / \cosh (t))),
$$

where $\arcsin (1 / \cosh (t))$ is the angle between vertical line and the boundary of its $t$-neighborhood. Using this, Calegari [4] obtains a formula of the volume of the piece $\mathcal{C}(l)$ with ortholength $l$, namely

$$
\begin{array}{r}
\frac{1}{2} \operatorname{vol}_{2 n-1}(\mathcal{C}(l))=\int_{0}^{l} \cosh ^{n-1}(t) V_{n-1}^{H}(l(l)) V_{n-1}^{S}(\arcsin (1 / \cosh (t))) \Omega_{n-1}^{-1} d t \\
+\int_{l}^{\infty} \cosh ^{n-1}(t)\left(V_{n-1}^{H}(l(l))-V_{n-1}^{H}\left(\iota_{\phi}(l)\right)\right) \\
\cdot V_{n-1}^{S}(\arcsin (1 / \cosh (t))) \Omega_{n-1}^{-1} d t .
\end{array}
$$

\subsection{The volume of Calegari's piece of dimension 3}

We are ready to give an explicit expression for the volume of a piece in dimension three. It is more convenient to work with Calegari's decomposition (compare [3, Section 4.2]) and we have to evaluate the following integral:

$$
\begin{aligned}
\frac{1}{2} \operatorname{vol}_{5}(\mathcal{C}(l))=\int_{0}^{l} \cosh ^{2}(t) V_{2}^{H}(\iota(l)) & V_{2}^{S}(\arcsin (1 / \cosh (t))) \Omega_{2}^{-1} d t \\
& +\int_{l}^{\infty} \cosh ^{2}(t)\left(V_{2}^{H}(\iota(l))-V_{2}^{H}\left(\iota_{\phi}(l)\right)\right) \\
& \cdot V_{2}^{S}(\arcsin (1 / \cosh (t))) \Omega_{2}^{-1} d t .
\end{aligned}
$$

By easy computation, we have

$$
\begin{aligned}
V_{2}^{H}(r) & =2 \pi(\cosh (r)-1), \\
V_{2}^{S}(r) & =2 \pi(1-\cos (r)) .
\end{aligned}
$$


Then by substitution, we have

$$
V_{2}^{S}(\arcsin (1 / \cosh (t)))=2 \pi\left(1-\sqrt{1-1 / \cosh ^{2}(t)}\right)=2 \pi(1-\tanh (t)) .
$$

Now we compute the first term of (3),

$$
\begin{aligned}
\int_{0}^{l} \cosh ^{2}(t) V_{2}^{H}(\iota(l)) V_{2}^{S}(\arcsin (1 / & \cosh (t))) \Omega_{2}^{-1} d t \\
& \left.=\pi \int_{0}^{l} \cosh ^{2}(t)(\cosh (l(l))-1)\right)(1-\tanh (t)) d t \\
& =\pi(\cosh (l(l))-1) \int_{0}^{l} \cosh ^{2}(t)(1-\tanh (t)) d t \\
& =\frac{\pi}{2}(\cosh (\iota(l))-1) \int_{0}^{l}\left(e^{-2 t}+1\right) d t \\
& =\frac{\pi}{4}(\cosh (l(l))-1)\left(-e^{2 l}+2 l+1\right) .
\end{aligned}
$$

We calculate the second term of (3) using the equations $\cosh \left(\iota_{\phi}(l)\right)=\tanh (t) / \tanh (l)$ and $\cosh (l(l))=1 / \tanh (l)$ :

$$
\begin{aligned}
\int_{l}^{\infty} \cosh ^{2}(t)\left(V_{2}^{H}(l(l))\right. & \left.-V_{2}^{H}\left(\iota_{\phi}(l)\right)\right) V_{2}^{S}(\arcsin (1 / \cosh (t))) \Omega_{2}^{-1} d t \\
& =\pi \int_{l}^{\infty} \cosh ^{2}(t)\left(\cosh (\iota(l))-\frac{\tanh (t)}{\tanh (l)}\right)(1-\tanh (t)) d t \\
& =\pi \cosh (\iota(l)) \int_{l}^{\infty} \cosh ^{2}(t)+\sinh ^{2}(t)-2 \cosh (t) \sinh (t) d t \\
& =\pi \cosh (\iota(l)) \int_{l}^{\infty} e^{-2 t} d t \\
& =\frac{\pi}{2} \cosh (\iota(l)) e^{-2 l} .
\end{aligned}
$$

Therefore, we have

$$
\begin{aligned}
\frac{\operatorname{vol}_{5}(\mathcal{C}(l))}{2} & =\frac{\pi}{4}(\cosh (\iota(l))-1)\left(-e^{2 l}+2 l+1\right)+\frac{\pi}{2} \cosh (\iota(l)) e^{-2 l} \\
& =\frac{\pi}{4}\left(\left(\frac{1}{\tanh (l)}+1\right) e^{-2 l}+\left(\frac{1}{\tanh (l)}-1\right)(2 l+1)\right)
\end{aligned}
$$

if and only if $\frac{\operatorname{vol}_{5}(\mathcal{C}(l))}{2}=\frac{\pi(l+1)}{e^{2 l}-1}$, and this completes the proof of Theorem 2. 


\section{A closed formula in odd dimensions}

For completeness we include a proof that the volume of each piece $\mathcal{C}(l)$ is computable, that is, can be expressed in terms of standard functions and the dilogarithm.

Proposition $6 \operatorname{vol}_{2 n-1}(\mathcal{C}(l))$ can be expressed in terms of elementary functions if the dimension $n$ is odd.

Proof Since $V_{n-1}^{H}(l(l))$ and $\Omega_{n-1}^{-1}$ do not depend on $t$, it suffices to consider

$$
\begin{gathered}
\int_{0}^{l} \cosh ^{n-1} V_{n-1}^{S}(\arcsin (1 / \cosh (t))) d t \\
\int_{l}^{\infty} \cosh ^{n-1}(t)\left(-V_{n-1}^{H}\left(\iota_{\phi}(l)\right)\right) V_{n-1}^{S}(\arcsin (1 / \cosh (t))) d t .
\end{gathered}
$$

Integrating by parts yields $V_{n-1}^{S}(r)=\sum_{i=0}^{n-1} q_{i} \sin (r)^{i} \cos (r)^{n-1-i}$ for some $q_{i} \in \mathbb{Q}$. Since $\cos (\arcsin (1 / \cosh (t)))=1 / \tanh (t)$, we see that the integrand of (4) is a rational function of $e^{t}$, and hence the integral (4) is computable. For (5), we need the assumption that $n$ is odd. Let $n=2 m+1$, then by partial integration,

$$
\begin{aligned}
V_{n-1}^{H}(r)=V_{2 m}^{H}(r) & =\int_{0}^{r} \sinh ^{2 m-1}(t) d t \\
& =\left[\cosh (t)\left(1-\cosh ^{2}(t)\right)^{m-1}\right]_{0}^{r}-(2 m-2)\left(V_{2 m-2}^{H}+V_{2} m^{H}\right) .
\end{aligned}
$$

As we see in Section 8.1.1, $\cosh \left(\iota_{\phi}(l)\right)=\tanh (t) / \tanh (l)$. (Note that if $n$ is even, the integrand contains $\sinh \left(\iota_{\phi}(l)\right)$ and we need square root for $\left.\sinh \left(\iota_{\phi}(l)\right)\right)$. Hence if $n$ is odd, the integrand of (5) is also a rational function of $e^{t}$. Hence, the integral (5) can be evaluated in terms of usual functions and dilogarithms, just as Bridgeman-Kahn's integral when $n$ is even, but now provided that $n$ is odd.

In the light of this proposition, we illustrate the difficulty involved in directly comparing the volumes $\operatorname{vol}_{2 n-1}(\mathcal{B}(l))$ and $\operatorname{vol}_{2 n-1}(\mathcal{C}(l))$ in dimensions $n \geq 3$. We consider just the case of $\operatorname{vol}_{5}(\mathcal{B}(l))$ for which one has the following formula from [3, Section 4.2]:

$$
\operatorname{vol}_{5}(\mathcal{B}(l))=2 \int_{0}^{1} \frac{M_{3}\left(\sqrt{\left(a^{2}-r^{2}\right) /\left(1-r^{2}\right)}\right)}{\sqrt{1-r^{2}}} d r
$$

where

$$
M_{3}(x)=\frac{2}{x^{2}-1}(1-\log (2))-\frac{1}{2 x}\left(\frac{x-1}{x+1}\right) \log (x-1)+\frac{1}{2 x}\left(\frac{x+1}{x-1}\right) \log (x+1) .
$$


By easy computation,

$$
\operatorname{vol}_{5}(\mathcal{B}(l))=\frac{\pi(1-\log (2))}{a^{2}-1}+2 \int_{0}^{1} \frac{N_{3}\left(\sqrt{\left(a^{2}-r^{2}\right) /\left(1-r^{2}\right)}\right)}{\sqrt{1-r^{2}}} d r
$$

where

$$
N_{3}(x)=-\frac{1}{2 x}\left(\frac{x-1}{x+1}\right) \log (x-1)+\frac{1}{2 x}\left(\frac{x+1}{x-1}\right) \log (x+1) .
$$

By changing variables, we have

$$
\begin{aligned}
& \operatorname{vol}_{5}(\mathcal{B}(l))=\frac{\pi(1-\log (2))}{e^{2 l}-1} \\
& \quad+\sqrt{e^{2 l}-1} \int_{0}^{\infty}\left\{\frac{\log \left(e^{l} \cosh x+1\right)}{\left(e^{l} \cosh x-1\right)^{2}}-\frac{\log \left(e^{l} \cosh x-1\right)}{\left(e^{l} \cosh x+1\right)^{2}}\right\} d x .
\end{aligned}
$$

Remark We substitute $a=e^{l}$, because $a \cosh x$ might be confused with $\operatorname{arccosh} x$. Now we have the following as a corollary of Theorems 1 and 2.

\section{Corollary 7}

$$
\int_{0}^{\infty}\left\{\frac{\log \left(e^{l} \cosh x+1\right)}{\left(e^{l} \cosh x-1\right)^{2}}-\frac{\log \left(e^{l} \cosh x-1\right)}{\left(e^{l} \cosh x+1\right)^{2}}\right\} d x=\frac{\pi}{\sqrt{e^{2 l}-1}}(l+\log (2)) .
$$

We note that a simple change of variable $u=\cosh x$ yields an integral which one might be tempted to evaluate using contour integration in $\mathbb{C}$. However, since the derivative of $\cosh x$ is $\sinh x$ there is a factor $\left(u^{2}-1\right)^{-1 / 2}$ which seems difficult to deal with using standard techniques.

\section{References}

[1] A Basmajian, The orthogonal spectrum of a hyperbolic manifold, Amer. J. Math. 115 (1993) 1139-1159 MR1246187

[2] M Bridgeman, Orthospectra of geodesic laminations and dilogarithm identities on moduli space, Geom. Topol. 15 (2011) 707-733 MR2800364

[3] M Bridgeman, J Kahn, Hyperbolic volume of manifolds with geodesic boundary and orthospectra, Geom. Funct. Anal. 20 (2010) 1210-1230 MR2746952

[4] D Calegari, Chimneys, leopard spots and the identities of Basmajian and Bridgeman, Algebr. Geom. Topol. 10 (2010) 1857-1863 MR2684984

[5] D Calegari, Bridgeman's orthospectrum identity, Topology Proc. 38 (2011) 173-179 MR2680162 
[6] W Fenchel, Elementary geometry in hyperbolic space, de Gruyter Studies in Mathematics 11, Walter de Gruyter \& Co, Berlin (1989) MR1004006

[7] J G Ratcliffe, Foundations of hyperbolic manifolds, 2nd edition, Graduate Texts in Mathematics 149, Springer, New York (2006) MR2249478

Department of Mathematical and Computing Sciences, Tokyo Institute of Technology O-okayama, Meguro-ku, Tokyo 152-8552, Japan

Institut Fourier

100 rue des maths, BP 74, 38402 St Martin d'Hères, France

masai9@is.titech.ac.jp, mcshane@ujf-grenoble.fr

http://www-fourier.ujf-grenoble.fr/ mcshane/

Received: 8 April 2013 Revised: 2 May 2013 